\title{
Evaluación del grado de cumplimiento de las BPM en la industria cervecera artesanal de la Región de los Ríos
}

\author{
Assessment of the degree of compliance with GMP \\ in the Craft Brewery Industry of the Region of Los Ríos
}

\author{
Díaz, A. ${ }^{a}$, Carrillo, B. ${ }^{a^{*}}$ \\ ${ }^{a}$ Instituto de Ciencia y Tecnología de los Alimentos, Facultad de Ciencias Agrarias, \\ Universidad Austral de Chile. Valdivia, Chile. Casilla 567. Valdivia..
}

\begin{tabular}{l} 
A R T I C L E I N F O \\
\hline Article history: \\
Received 05.08.2016 \\
Accepted 13.12.2016 \\
\hline Keywords: \\
Beer \\
Craft beer \\
Check list \\
\hline Original Research Article, \\
Food Science \\
\hline *Corresponding author: \\
Aliciela Díaz, Bernardo Carrillo \\
E-mail address: \\
bcarrill@uach.cl
\end{tabular}

\section{A B S T R A C T}

The compliance with Good Manufacturing Practices (GMP) was assessed in 73\% of traditional breweries in the Region of Los Ríos, most of them (84\%) members of the "Unión de Cerveceros Los Ríos (UCR)", without previous records.

A "Check list" containing the guidelines of the Chilean Ministry of Health to evaluate food industries and allow their operation was used. This guideline includes: installation, cleaning, sanitation, pest control, personnel hygiene, training, raw materials, processes and finished products; it was only used as a reference since the brewing industry must comply with the regulations set up by the Agricultural and Livestock Service. In addition, an additional guideline was included to report geographical location, production and sales, among others; in order to obtain a general characterization of the industry in the Region.

Out of the surveyed industries, $12(55 \%)$ are located in urban areas, and $10(45 \%)$ in rural areas. The majority are Micro Breweries (63\%), divided mainly in Micro 3 (36\%) and Micro 1 (27\%) according to the classification of the Tax Administration (SII) service in Chile.

Only $18 \%$ of the industries comply with GMP in $70 \%$ or more, which highlights the importance of supporting initiatives that are currently being developed in these industries in the Region of Los Ríos, to improve production and consolidate market presence. The best evaluated item was "finished products and processes" (73\%), adequate storage conditions of the product was highlighted, and the worst "training" (10.2\%), mainly in hygienic food handling, and equipment cleaning and sanitation.

\section{RESUMEN}

Se evaluó grado de cumplimiento de Buenas Prácticas de Manufactura (BPM) en el 73\% de cervecerías artesanales de la Región de Los Ríos, la mayoría (84\%) miembros de "Unión de Cerveceros de Los Ríos (UCR)", antecedentes sin información previa. La evaluación se realizó aplicando un "Check list" utilizado por el Ministerio de Salud de Chile para evaluar industrias alimentarias y permitir su funcionamiento. Esta pauta contempla: instalación, limpieza, sanitización, control de plagas, higiene del personal, capacitación, materias primas, procesos y productos terminados; sólo se utilizó como referencia, ya que la industria cervecera se rige por normativa fiscalizada por Servicio Agrícola y Ganadero. Además, se incorporó una pauta que informaría respecto a ubicación geográfica, producción, ventas, entre otras; para obtener una caracterización general de esta industria en la región.

De las industrias encuestadas, 12 (55\%) están en zona urbana, y 10 (45\%) en zona rural. La mayoría, correspondería a Micro Cervecerías (63\%), divididas en Micro 3 (36\%) y Micro 1 (27\%), principalmente; según clasificación del Servicio de Impuestos Internos (SII) de Chile.

Por otra parte, sólo 18\% cumplen con BPM, arrojando un cumplimiento igual o superior al 70\%, por lo que se estima importante apoyar iniciativas que actualmente se desarrollan en estas industrias de la región de Los Ríos, mejorando producción y afianzando participación en el mercado. El ítem mejor evaluado fue "procesos y productos terminados" (73\%), destacando adecuadas condiciones de almacenamiento del producto, y el peor "capacitación" (10,2\%), principalmente en manipulación higiénica de alimentos, y limpieza y sanitización de equipos.

Palabras clave: Cerveza, cerveza artesanal, check list.

\section{INTRODUCCIÓN}

La cerveza, a pesar de ser una bebida microbiológicamente estable, por la presencia de alcohol, com- puestos amargos del lúpulo, contenido de dióxido de carbono, bajo pH $(3,8-4,7)$ y reducida concentración de oxígeno, además de ser un medio pobre de nutrientes los que son casi agotados por la fermentación de 
la levadura, puede contener microorganismos como Lactobacillus, Pediococcus, Pectinatus y Megasphaera, particularmente dañinos en términos de frecuencia de incidentes de descomposición y efectos negativos en los perfiles de sabor de la cerveza (Suzuki, 2011). Por ejemplo, especies del género Lactobacillus provocan una alta turbidez, aspecto brumoso, sabores desagradables y un alto nivel de diacetilo en cervezas, por lo tanto, es necesario adoptar medidas adecuadas para reducir la incidencia de estas bacterias en el deterioro; dado que la contaminación es causada en las últimas etapas de procesamiento, las pérdidas son altas (Paradh et al., 2011). La manera más efectiva de prevenir tal deterioro de la cerveza es a través del control de la contaminación, con la limpieza y el adecuado saneamiento (Manzano et al., 2011).

A causa de la aparición de estos problemas de deterioro en la cerveza y al mismo tiempo para asegurar la inocuidad del producto, tanto a nivel industrial como artesanal, es que tanto en España, EEUU y Argentina, por nombrar algunos países, se ha implementado el sistema de aseguramiento de calidad con el Análisis de Peligros y Puntos Críticos de Control (APPCC o su sigla en inglés HACCP) (Cerveceros de España, 2005; Ministerio de Agricultura, Ganadería y Pesca, 2016).

En Chile, según la Norma Chilena 2861, uno de los pre-requisitos para la implementación del HACCP es el cumplimiento de las Buenas Prácticas de Manufactura (BPM), las cuales deben estar firmemente establecidas $\mathrm{y}$ en pleno funcionamiento, haberse verificado adecuadamente, facilitando la aplicación de dicho sistema de aseguramiento de calidad (Chile, Instituto Nacional de Normalización (INN), 2011). Las BPM comprenden las prácticas aplicadas de higiene, orientadas a asegurar las condiciones básicas y favorables necesarias para la producción de alimentos inocuos y que están en conformidad con los códigos, normas, leyes, y reglamentos referentes a la producción, elaboración, manipulación, etiquetado, almacenamiento y venta de ellos (Chile, Ministerio de Salud (MINSAL), 2015).

De acuerdo a lo anterior, en Chile y en la Región de Los Ríos, las industrias cerveceras artesanales deberían cumplir al menos con los requisitos de BPM, pese a que para su normal funcionamiento éstas no requieren de una autorización por parte de la Autoridad Sanitaria (MINSAL), como es el caso en la industria alimentaria, sino que sólo un informe sanitario que se lleva a cabo con la Secretaría Regional Ministerial de Salud, estar registradas como cervecería en el Servicio Agrícola y Ganadero (SAG) y así también tener registrados sus productos y cumplir además con la normativa vigente (Ley 18.455 y Decreto $\mathrm{N}^{\circ} 78$ ), donde su cumplimiento es fiscalizado por el SAG (Chile, Ministerio de Agricultura (MINAGRI), 2013).

Dada la importancia de la implementación de las BPM para la obtención de productos inocuos, y además considerando que a la fecha no existen antecedentes que caractericen a este sector productivo, es que resulta interesante evaluar el grado de cumplimiento de éstas en la Industria Cervecera Artesanal de la Región de Los Ríos, la mayoría miembros de la Unión de Cerveceros de Los Ríos (UCR).

\section{MATERIAL Y MÉTODO}

\section{Material}

Área de estudio. La presente investigación se llevó a cabo durante los meses de Octubre a Diciembre del año 2015, en las comunas de San José de la Mariquina, Los Lagos, Paillaco, Panguipulli, Valdivia y La Unión, XIV Región de Los Ríos, Chile.

Universo de estudio. Estuvo constituido por 30 cervecerías artesanales, registradas a diciembre de 2014 en el Servicio Nacional de Turismo de la Región de Los Ríos (Chile, Servicio Nacional de Turismo (SERNATUR), 2014).

Muestra. La muestra estuvo constituida por 22 cervecerías artesanales (Figura 1). Estas constituyen el 84\% de las 26 cervecerías pertenecientes a UCR (Cuadro 1) y al 73\% del universo de cervecerías de la Región de Los Ríos. La selección de la muestra para fines de este estudio fue de carácter no probabilístico, ya que según Hernández et al. (2006), la elección de los elementos en este tipo de muestras no depende de la probabilidad, sino de las características de investigación o de quien

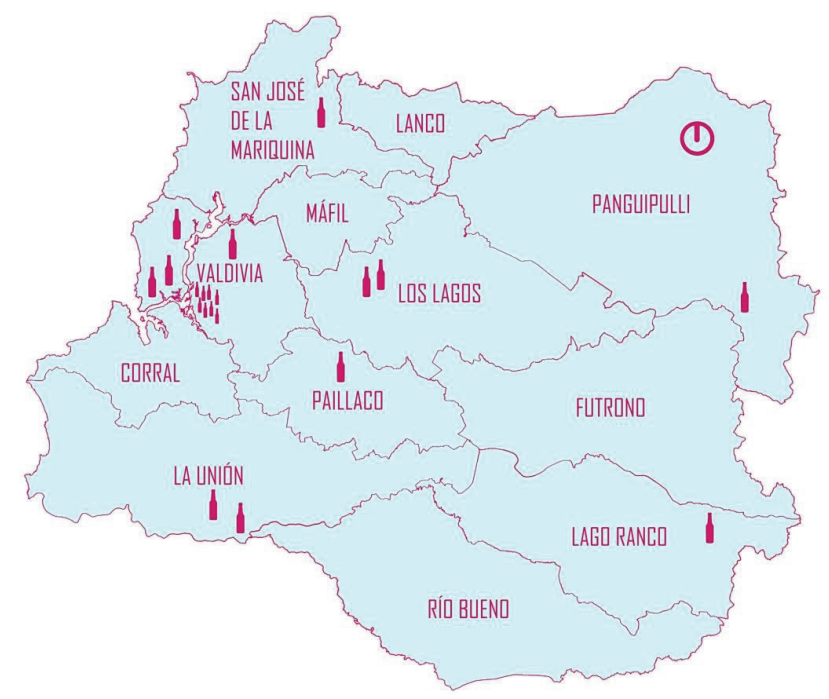

Figura 1. Ubicación de las cervecerías UCR estudiadas en la Región de Los Ríos.

Figure 1. Location of the UCR breweries studied in the Los Ríos Region. 
hace el muestreo. En este estudio, la muestra corresponde prácticamente a todas las cervecerías pertenecientes a la UCR, las que forman parte de un proyecto que busca el fortalecimiento de las cervecerías de la región, y que decidieron participar voluntariamente en el estudio.

\section{Método}

Se utilizó una pauta de evaluación extraída y utilizada por el Ministerio de Salud de Chile, en el marco de su política de inocuidad de los alimentos. La pau- ta denominada Lista de Chequeo- Buenas Prácticas de Manufactura fue sometida primeramente a una prueba piloto, de acuerdo a lo que sugiere Hernández et al. (2006), para este tipo de estudio. Ésta consiste en administrar el instrumento a una pequeña muestra, cuyos resultados se utilizan para calcular confiabilidad inicial, y de ser posible, la validez del instrumento. Es por ello que la pauta fue probada inicialmente en tres cervecerías, y como resultado de ello se mantuvo casi la totalidad de los parámetros, a excepción del número 30 incluido en el ítem Materias Primas, basado en el Art. 28 del Reglamento Sanitario de los Alimentos (RSA), el

Cuadro 1. Establecimientos de la Unión de Cerveceros de Los Ríos (UCR). 2015

Table 1. Establishments members of the "Unión de Cerveceros de Los Ríos (UCR)". 2015

\begin{tabular}{|c|c|c|}
\hline Establecimientos & Ubicación & Comuna \\
\hline A & Calcurrupe Alto s/n & Lago Ranco \\
\hline B & Camino Internacional Km 77-Puerto Fuy-Panguipulli-Valdivia & Panguipulli \\
\hline $\mathrm{C}$ & Cudico Km 10 & La Unión \\
\hline $\mathrm{D}$ & Rafael Azócar N¹012 & La Unión \\
\hline $\mathrm{E}$ & Martínez de Rosas N³311 & Valdivia \\
\hline $\mathrm{F}^{*}$ & $\mathrm{~s} / \mathrm{i}$ & $\mathrm{s} / \mathrm{i}$ \\
\hline G & Km 8 Ruta T- 350 s/n & Valdivia \\
\hline $\mathrm{H}$ & Las Canteras $\mathrm{N}^{\circ} 15$. Ruta $\mathrm{T}-350$ & Valdivia \\
\hline I & Vicuña Mackenna $\mathrm{N}^{\circ} 1281$ & Paillaco \\
\hline $\mathrm{J}$ & Camino a Panguipulli. Los Lagos Km 2 & Panguipulli \\
\hline $\mathrm{K}$ & Lote 1. Hijuela 1.Lote B el cuadro B. & Los Lagos \\
\hline $\mathrm{L}$ & Mariquina 045 & San José de la Mariquina \\
\hline M & Santa Elvira, Km 5 & Valdivia \\
\hline $\mathrm{N}^{*}$ & $\mathrm{~s} / \mathrm{i}$ & $\mathrm{s} / \mathrm{i}$ \\
\hline 0 & Eugenio Matte $\mathrm{N}^{\circ} 92$ & Valdivia \\
\hline $\mathrm{P}$ & Villa Bosques de Entre Ríos. Calle Volcán Michimahuida №4669 & Valdivia \\
\hline Q & Llancahue s/n & Valdivia \\
\hline $\mathrm{R}^{*}$ & $\mathrm{~s} / \mathrm{i}$ & $\mathrm{s} / \mathrm{i}$ \\
\hline S & Cayumapu Km 25 Norte & Valdivia \\
\hline $\mathrm{T}$ & Domeyko $N^{\circ} 105$ & Valdivia \\
\hline $\mathrm{U}^{*}$ & $\mathrm{~s} / \mathrm{i}$ & $\mathrm{s} / \mathrm{i}$ \\
\hline $\mathrm{V}$ & Camino Angachilla Km 6 & Valdivia \\
\hline $\mathrm{W}$ & Nueva Uno $N^{\circ} 950$. Villa Rocura. Las Ánimas & Valdivia \\
\hline $\mathrm{X}$ & Holanda $N^{\circ} 1761$ & Valdivia \\
\hline Y & Punucapa s/n & Valdivia \\
\hline $\mathrm{AB}$ & San Luis $N^{\circ} 1045$ & Valdivia \\
\hline
\end{tabular}

* Industria no visitada. 
cual hace referencia al uso de hielo que tiene contacto directo con alimentos durante la elaboración, y que en este caso no aplicaba.

La pauta modificada se aplicó en cada una de las cervecerías, evaluando los ítems: instalaciones, limpieza y sanitización, control de plagas, higiene del personal, capacitación, materias primas, procesos y productos terminados. En cada ítem existía una serie de parámetros que fueron evaluados, los que se muestran resumidos en el Cuadro 2. Cada instalación logró un puntaje o grado de cumplimiento respecto de un total posible de alcanzar, y cuando no se cumplía con algún parámetro se registró la observación correspondiente. Adicionalmente, posterior a cada visita se procedió a elaborar un informe, el cual detallaba lo evaluado, con observaciones y recomendaciones para que una vez co- rregidas, se alcance el puntaje máximo en cada ítem. El informe fue enviado individualmente a los representantes de las cervecerías.

De acuerdo al Instructivo de Aplicación de la Lista de Chequeo BPM del 2015 (Chile, Ministerio de Salud (MINSAL), 2015), la designación del puntaje para cada parámetro es de 2 cuando hay cumplimiento total, 1 cuando es parcial, 0 cuando no hay cumplimiento y N/A cuando el parámetro no aplica al establecimiento. El puntaje total obtenido por el establecimiento debe ser igual o superior al $70 \%$, habiendo necesariamente cumplido con los factores críticos señalados en el Instructivo, para cumplir con las BPM; sin embargo, todo establecimiento que no logre el $100 \%$ de cumplimiento genera un proceso de Acta de Fiscalización con derivación a la Fiscalía Sanitaria, de acuerdo a lo señalado por

Cuadro 2. Parámetros evaluados mediante el Check List.

Table 2. Parameters evaluated using the Checklist.

\begin{tabular}{|c|c|c|c|}
\hline No. Item & Descripción & No. Item & Descripción \\
\hline 1 & Piso y paredes & 21 & Programa de control de plagas \\
\hline 2 & Cielos y estructuras elevadas & 22 & $\begin{array}{l}\text { Zona de almacenamiento de desechos y receptá- } \\
\text { culos }\end{array}$ \\
\hline 3 & Ventanas y otras aberturas & 23 & $\begin{array}{l}\text { Autorización Sanitaria de empresa a cargo del } \\
\text { control de plagas }\end{array}$ \\
\hline 4 & Estructuras auxiliares & 24 & Programa y registros de higiene del personal \\
\hline 5 & Superficies y equipos de trabajo & 25 & $\begin{array}{l}\text { Medidas preventivas frente a manipuladores en- } \\
\text { fermos }\end{array}$ \\
\hline 6 & Evacuación de aguas residuales & 26 & $\begin{array}{l}\text { Limpieza del personal y ropa acorde a sus fun- } \\
\text { ciones }\end{array}$ \\
\hline 7 & $\begin{array}{l}\text { Mantenciones preventivas de instalaciones, } \\
\text { equipos y utensilios }\end{array}$ & 27 & $\begin{array}{l}\text { Programa de capacitación en manipulación hi- } \\
\text { giénica de alimentos }\end{array}$ \\
\hline 8 & Agua potable & 28 & $\begin{array}{l}\text { Programa de capacitación en técnicas de limpie- } \\
\text { za }\end{array}$ \\
\hline 9 & Distribución de agua & 29 & Materias primas utilizadas \\
\hline 10 & Servicios higiénicos & 30 & $\begin{array}{l}\text { Hielo para proceso de elaboración (no aplicaba a } \\
\text { las cervecerías) }\end{array}$ \\
\hline 11 & Ventilación & 31 & Registros de control de materias primas \\
\hline 12 & Iluminación & 32 & Especificaciones escritas de materias primas \\
\hline 13 & Equipos de iluminación & 33 & Almacenamiento de materias primas \\
\hline 14 & Lugar de disposición de desechos & 34 & Flujo del personal, vehículos y materias primas \\
\hline 15 & Medidas para disposición y retiro de desechos & 35 & Procedimientos escritos de los procesos \\
\hline 16 & Equipos de frío & 36 & Almacenamiento de los productos \\
\hline 17 & Programa de limpieza y sanitización & 37 & Distribución de productos terminados \\
\hline 18 & Registro del programa de limpieza y sanitización & 38 & Material para envasar los productos \\
\hline 19 & $\begin{array}{l}\text { Medidas que eviten contaminación de equipos } \\
\text { posterior a su limpieza y desinfección }\end{array}$ & 39 & Etiquetado de los productos \\
\hline 20 & Almacenamiento de productos químicos & & \\
\hline
\end{tabular}


el Departamento de Acción Sanitaria de la Secretaría Regional Ministerial de Salud de la Región de Los Ríos.

También, se elaboró y aplicó una pauta para identificar y caracterizar a modo general las cervecerías encuestadas (Cuadro 3).
Análisis estadístico. Los datos se trabajaron usando estadística descriptiva, con tablas de distribución de frecuencias y gráficos para resumir la información obtenida, de acuerdo a lo sugerido por Di Renzo et al. (2005), para este tipo de estudios.

Cuadro 3. Pauta adicional a la Lista de Chequeo BPM.

Table 3. Additional guideline added to the GMP Checklist GMP.

B) Información general del establecimiento: Cervecería "A, B, C, etc."

Razón Social Propietario:

Rut.:

Categoría Industrial (Según nivel de ventas UF/año del establecimiento, conforme los antecedentes proporcionados por la empresa)

\begin{tabular}{|c|c|c|}
\hline Categoría Industrial & Ventas (UF/año) & \\
\hline Sin ventas & & $\begin{array}{l}\text { Corresponde a contribuyentes cuya información tributaria } \\
\text { declarada, no permite determinar un monto estimado de ventas. }\end{array}$ \\
\hline Micro 1 & & 0,01 UF a $200 \mathrm{UF}$ \\
\hline Micro 2 & & 200,01 UF a 600 UF \\
\hline Micro 3 & & $600,01 \mathrm{UF}$ a $2.400 \mathrm{UF}$ \\
\hline Pequeña 1 & & $2.400,01$ UF a 5.000 UF \\
\hline Pequeña 2 & & $5.000,01$ UF a 10.000 UF \\
\hline Pequeña 3 & & $10.000,01 \mathrm{UF}$ a $25.000 \mathrm{UF}$ \\
\hline Mediana 1 & & $25.000,01 \mathrm{UF}$ a $50.000 \mathrm{UF}$ \\
\hline Mediana 2 & & $50.000,01$ UF a 100.000 UF \\
\hline Grande 1 & & $100.000,01$ UF a 200.000 UF \\
\hline Grande 2 & & $200.000,01$ UF a 600.000 UF \\
\hline Grande 3 & & $600.000,01$ UF a 1.000 .000 UF \\
\hline Grande 4 & & Más de 1.000.000 UF \\
\hline
\end{tabular}

Destino específico de la producción:

Estacionalidad de la producción:

C) Otros antecedentes:

\author{
Variedad (es) de cerveza: \\ Empresa proveedora de Malta: \\ Variedades de Malta: \\ Requerimiento de malta mensual (kg): \\ Origen de lúpulo: \\ Tipo de levadura (Ale o Lager): \\ Medio de hidratación de levadura: \\ Origen del agua para elaboración y otros usos: \\ Uso de agentes clarificantes de cerveza: \\ Uso de $\mathrm{CO}_{2}$ (carbonatación artificial) y/o azúcares para fermentación:
}




\section{RESULTADOS Y DISCUSIÓN}

\section{Caracterización de los establecimientos}

Se estableció que 12 cervecerías (55\%) están ubicadas en el área urbana, la mayoría de éstas en la comuna de Valdivia (67\%), y las restantes en las comunas de San José de la Mariquina, Paillaco y La Unión (33\%). Por otra parte, 10 cervecerías (45\%) estaban alejadas del área urbana, cerca de carreteras o incluso en sectores rurales de difícil acceso, como la cervecería A ubicada en la comuna de Lago Ranco y C ubicada en La Unión. En esta última, se pretende potenciar la venta de cerveza con el turismo rural, en el que ya se ha incursionado de manera modesta. A mayor escala, la cervecería B cuenta con un restaurant, formando parte de un complejo turístico. Otras iniciativas similares son I y K, las que, en un área contigua a la cervecería cuentan con un restaurant.

Entonces, una de las alternativas interesantes en el desarrollo de este rubro parece ser la producción de cerveza asociada al turismo y gastronomía, lo que coincide con lo señalado por Chile, Asociación de Productores de Cerveza de Chile (ACECHI), (2016).

Cabe señalar que la mayoría de las empresas, como se muestra en la Figura 2, no contrataba operarios, lo que puede ser una desventaja a la hora de aumentar los volúmenes de producción, porque habrá necesidad de mano de obra debidamente entrenada. Sin embargo, hay ocho cervecerías (36\%) que tienen a lo menos, un operario contratado durante todo el año, ya que por su volumen de producción es necesaria mayor mano de obra, (ej. Cervecería X y M). La mitad de las empresas no contaban con operarios externos, sino que son sus dueños los "maestros cerveceros" (50\%). Por otra parte, tres cervecerías (14\%) en épocas de mayor producción, han requerido de a lo menos un operario externo que trabaje en la elaboración de cerveza artesanal en ese período.
Estacionalidad de la producción. Dos cervecerías, $\mathrm{Hy} \mathrm{AB}$ por conveniencia, producen sólo estacionalmente. Éstas elaboran de Noviembre a Marzo, la primera, y desde Marzo a Septiembre, la segunda, y en bajos volúmenes, comparado con otras cervecerías de la UCR, que elaboran mayores volúmenes, y que además producen durante todo el año.

Destino de la producción. Estas cervecerías tienen diversos puntos de venta, los que incluyen restaurantes, y ferias que se realizan en algunos meses del año, tanto en Valdivia como en las demás regiones del país. En algunos casos, las cervezas se distribuyen principalmente en Valdivia, Temuco, Concepción y Santiago, entre otras ciudades de Chile.

Variedades de cerveza producidas. Elaboran al menos dos variedades, negra y rubia, pero depende de cada empresa la cantidad de variedades a producir. Por ejemplo, hay empresas que están buscando las preferencias del mercado y producen seis o hasta siete variedades, para posteriormente dedicarse a las que prefiere más el consumidor.

Categoría Industrial según nivel de ventas (UF/año). Con la información recopilada, y considerando la clasificación que hace el Servicio de Impuestos Internos de Chile (SII) sobre las empresas según nivel de ventas ${ }^{1}$, la mayoría de las cervecerías estarían en la categoría industrial Micro 3 (36\%) y Micro 1 (27\%), como se muestra en la Figura 3. Un 14\% estaría en Micro 2, y en el mismo porcentaje no se determinó la categoría por falta de información, pero al realizar la visita en terreno, se apreció que éstas pueden incluirse en la categoría Micro o Pequeñas empresas, ya que sus volúmenes de producción mensuales son similares a las de otras

\footnotetext{
http://www.sii.cl/estadisticas/empresas_tamano_ventas.htm
}

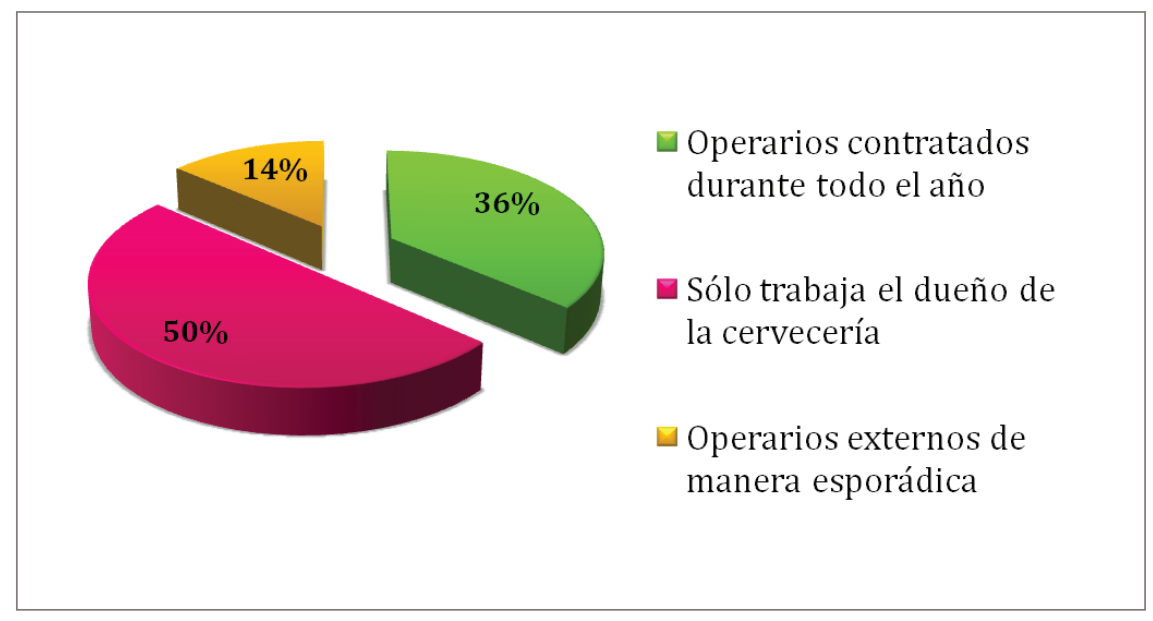

Figura 2. Tipo de operarios que trabajan en las cervecerías UCR encuestadas.

Figure 2. Type of operators working in the surveyed UCR breweries. 
industrias en esas categorías. En menor porcentaje se encuentra una cervecería en la categoría Pequeña 1 (5\%) y otra en Pequeña 2 (5\%).

\section{Grado de cumplimiento de las BPM}

Como resultado de la clasificación que tiene implementada la Autoridad Sanitaria según porcentajes de cumplimiento de las BPM, ningún establecimiento visitado clasificó en la categoría BPM1, es decir, el porcentaje de cumplimiento no sobrepasó el 90\%, el 18\% de los establecimientos está en BPM2, con porcentajes de cumplimiento entre 70 y $90 \%$, y la mayoría (82\%) se agrupa en la categoría BPM3, con porcentajes de cumplimiento menor a 70\%.

Estos datos indican que sólo las cervecerías BPM2 (18\%) fueron las que cumplieron con las BPM como se muestra en la Figura 4. Pese a ello, como estas industrias no se rigen por lo que exige la Autoridad Sanitaria a los efectos de autorizar su funcionamiento, más allá de los informes sanitarios que deben cumplir, no transgreden la normativa, ya que las cervecerías se rigen por otras nor-

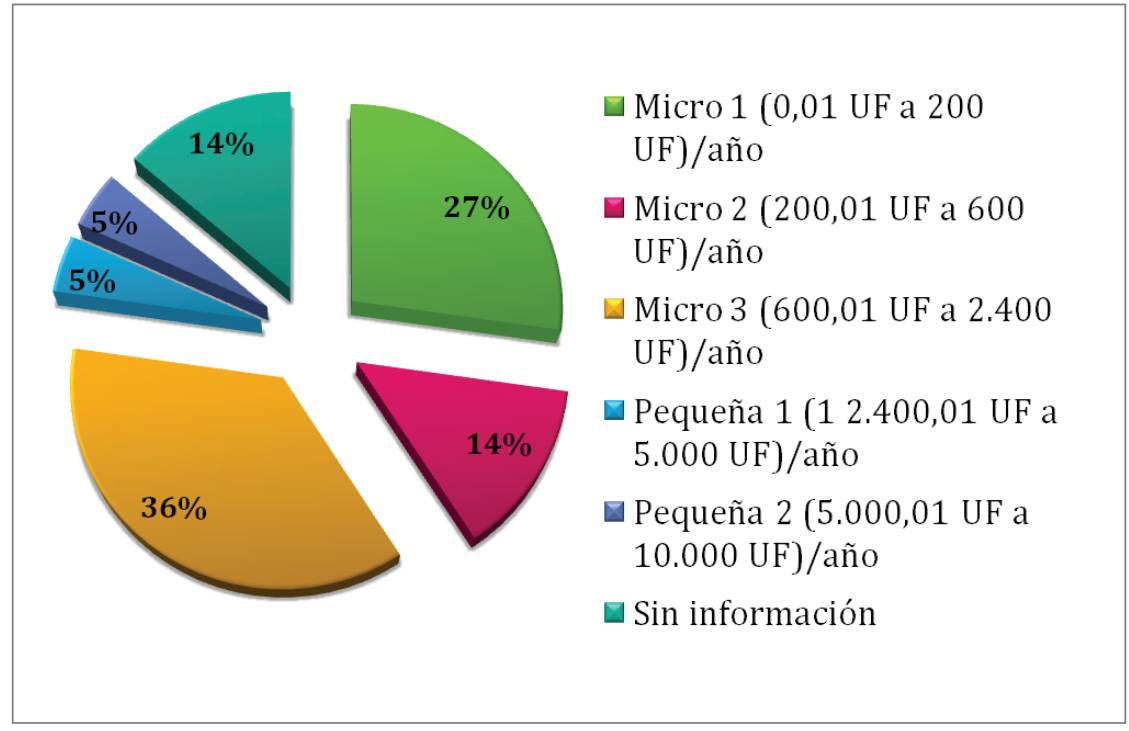

Figura 3. Agrupación de las empresas según Categoría Industrial establecida por el Servicio de Impuestos Internos (SII), 2015, según UF en ventas/año.

Figure 3. Classification of companies according to Industrial Category established by the Tax Administration service (SII), 2015, based on sales/year.

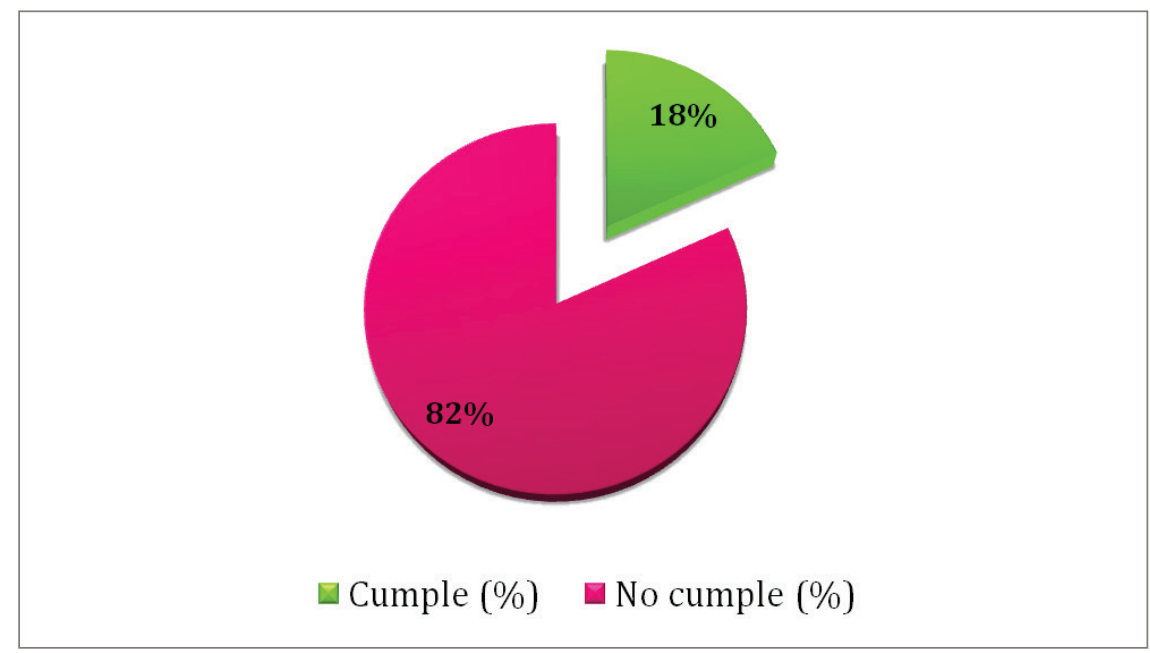

Figura 4. Porcentaje de cumplimiento BPM de las cervecerías del estudio.

Figure 4. Percentage of GMP compliance in the breweries under study. 
mativas, las cuales son fiscalizadas por el Servicio Agrícola y Ganadero. Sin embargo, estos antecedentes servirán para apoyar las iniciativas y proyectos que actualmente se están desarrollando a nivel de este tipo de industria en la región de Los Ríos, como por ejemplo el proyecto financiado con fondos regionales FIC 14-08: "Fortalecimiento Innovador Cerveceros - UACh" para mejorar su producción y afianzar su participación en el mercado.

Ítem limpieza y sanitización. En este ítem el porcentaje de cumplimiento promedio fue $41 \%$, y el puntaje máximo alcanzado por sólo cuatro establecimientos (A, I, K y S) (18\% del total de las cervecerías) fue de $63 \%$, según se observa en la Figura 5.

En la Figura 5 también se observa que las cervecerías L, Q T, W, Y y AB cumplen sólo con alrededor de un $25 \%$ en este ítem. Dentro de los parámetros que no cumplen están los referentes a la falta de un programa escrito de limpieza y sanitización y por ende falta de registros de dicho programa, y la inexistencia de un lugar de almacenamiento único para productos químicos. Es importante señalar que se deben estandarizar procesos y tener una rutina de limpieza que siempre genere óptimos resultados, ya que existen estudios que han evaluado la flora microbiana presente antes y después de procesos de higiene en cervecerías artesanales y se ha determinado que planes de saneamiento deficientes por el cervecero dio lugar a contaminación microbiana en la cervecería, demostrando así la importancia de la buena higiene que evite presencia de subproductos indeseables generados por microorganismos en el producto (Manzano et al., 2011).

Además, antes de implementar cualquier sistema de aseguramiento de calidad, es necesario cumplir con prerrequisitos, donde están incluidos por ejemplo,
"Procedimientos de limpieza y sanitización” y "Manejo de productos químicos", los cuales deben estar escritos y actualizados (CHILE, INN, 2011). En los registros se debiera especificar fecha, cómo y quién hizo la limpieza y/o sanitización, ya que es de suma importancia vigilar los Procedimientos Operativos de Limpieza y Sanitización para adaptar y/o corregirlos en función de los datos obtenidos a partir de la vigilancia (Chile, Sociedad Chilena de Microbiología e Higiene de los Alimentos (SOCHMHA) y Ministerio de Salud (MINSAL), 2004).

Ítem higiene del personal. Las cervecerías encuestadas tuvieron un bajo porcentaje de cumplimiento promedio en el ítem higiene del personal (44\%). Diez cervecerías (45\%) han logrado al menos el $50 \%$ de cumplimiento, mientras que 5 (23\%) no alcanzan el $20 \%$ de cumplimiento. A pesar de ello, un establecimiento (cervecería I, Figura 6) alcanzó un porcentaje notoriamente superior (83\%) al de sus pares. Éste no cumple el parámetro 24, el cual exige un programa de higiene del personal con sus respectivos registros; el establecimiento lo cumple parcialmente, dado que existe un programa, pero no se llevan registros ni documentación que aseguren la puesta en marcha de dicho programa y que demuestren eficacia de la vigilancia en el proceso. Al respecto, es importante señalar que, según la organización Cerveceros de España, cada prerrequisito lleva asociado una documentación que debe incluir el contenido de los planes y resultados de los controles (registros), con el fin de dejar constancia tanto de las intenciones y acciones a desarrollar, como de los resultados obtenidos de ellas (Cerveceros de España, 2005).

Para lograr que el personal manipulador tenga una conducta higiénica durante sus funciones, debe recibir

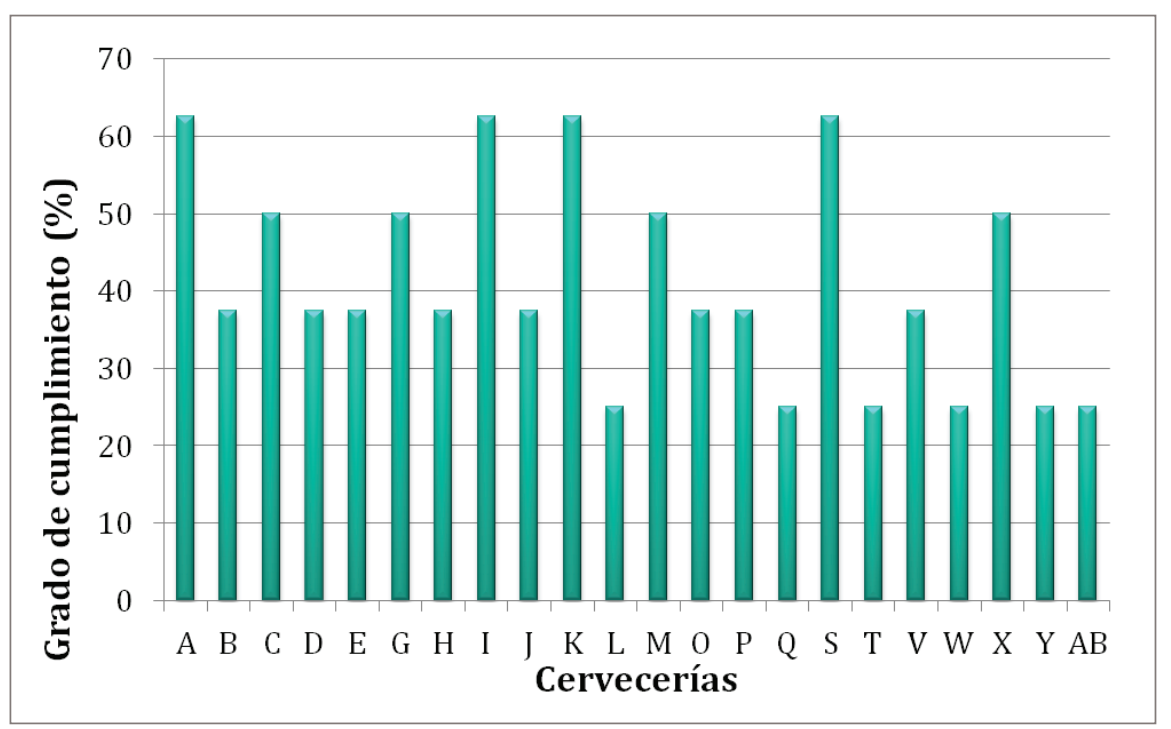

Figura 5. Grado de cumplimiento en el ítem limpieza y sanitización.

Figure 5. Degree of compliance with the item cleaning and sanitization. 
capacitaciones que le entreguen los conocimientos necesarios en esta materia. La importancia de la capacitación en manipulación de los alimentos, se debe a que el principal riesgo es microbiológico y depende directamente de la higiene del personal. Según los resultados del estudio de Brizio y Prentice (2014), donde se evaluó la contaminación cruzada entre manipuladores y productos avícolas de empresas de Brasil, se ha demostrado que los resultados microbiológicos con el uso de manos protegidas con guantes desechables fue significativamente mejor $(\mathrm{p}<0,05)$ comparado con el uso de manos desnudas y manos con guantes reutilizables.
Ítem capacitación. Este ítem permitió evaluar cuán capacitado está el personal dentro de la empresa, en materias como manipulación higiénica de los alimentos e higiene personal, y en técnicas de limpieza para el personal de aseo de la planta. El ítem se divide en dos parámetros, donde ningún establecimiento cumple totalmente con alguno de ellos.

Capacitación, fue el ítem con menor porcentaje de cumplimiento promedio (10\%) alcanzado por los establecimientos encuestados. En la Figura 7 aparece el grado de cumplimiento por parte de las cervecerías para este ítem, donde 16 (73\%) de éstas no cumplen

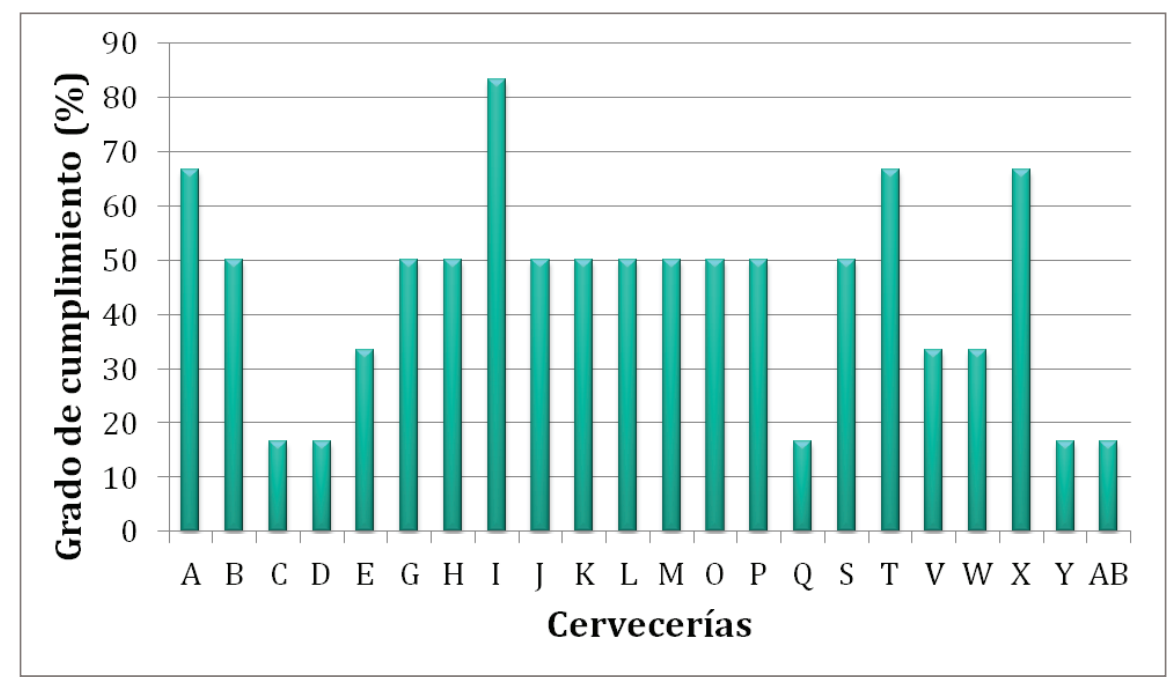

Figura 6. Grado de cumplimiento en el ítem higiene del personal. Figure 6. Degree of compliance with the item staff hygiene.

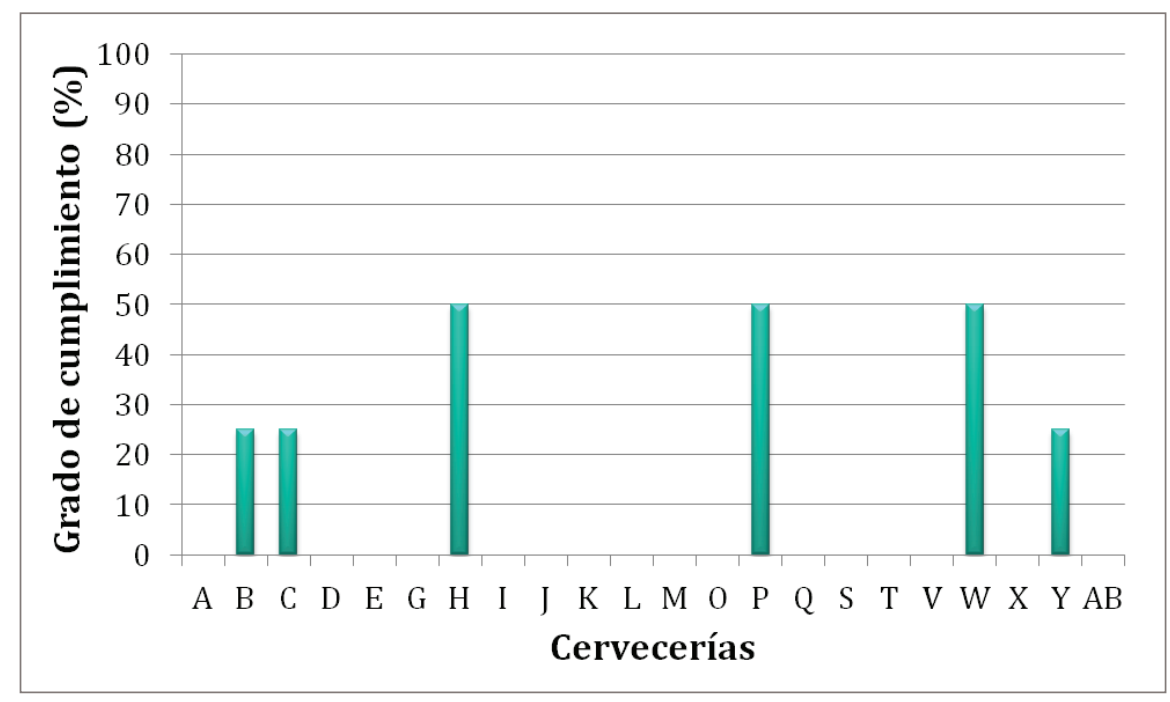

Figura 7. Grado de cumplimiento en el ítem capacitación.

Figure 7. Degree of compliance with the item training. 
con ninguno de los dos parámetros, es decir no tienen programas ni registros generados a partir de aquellos. Cabe señalar que si se tratase de industrias de alimentos, las que se rigen por el Reglamento Sanitario de los Alimentos y según el Art. 52 de éste, la dirección del establecimiento debe hacerse responsable que toda persona que trabaje en manipulación de alimentos reciba una instrucción adecuada y continua en esta materia y en higiene personal. En tanto para el personal a cargo del aseo se estipula en el Art. 41 del mismo reglamento que deberá estar capacitado en técnicas de limpieza.

En la misma Figura 7, se observa que las cervecerías $\mathrm{H}, \mathrm{P}$ y W, logran al menos el 50\% de cumplimiento, y las cervecerías B, C e Y presentaron alrededor de $25 \%$ de cumplimiento en este ítem.

Al comparar los resultados de estas industrias cerveceras artesanales con los resultados recientes de un estudio hecho en queserías artesanales de la Región de Los Ríos, éstos son muy similares. Según Mancilla (2014), las queserías que obtuvieron un puntaje de cumplimiento de las BPM menor a 70\% tienen como denominador común, la falta de capacitación de los operarios. En las cervecerías de este estudio hay falta de capacitación, incluso en aquellos que han superado el 70\% de cumplimiento de BPM. Las principales razones manifestadas por los maestros cerveceros durante la aplicación del "Check list", fue que han recibido capacitaciones en otras materias relacionadas directamente con el proceso de elaboración de cerveza y no en manipulación higiénica de los alimentos.

Ítem materias primas. Se revisó principalmente maltas, lúpulos y levaduras, ya que son las materias primas base del proceso de elaboración de cerveza. El agua no se abordó en este ítem, ya que su evaluación formó parte de otro ítem (ítem instalaciones).

Siendo este ítem uno de los que obtuvo mayor grado de cumplimiento de las BPM después del ítem procesos y productos terminados, con un cumplimiento promedio de $68 \%$. Ninguno de los establecimientos tiene menos de $50 \%$ de cumplimiento en el ítem, tal como se ve en la Figura 8.

Según la Brewers Association of the United States (Asociación de Cerveceros de los Estados Unidos²), en la sección proceso y controles del "Check list" de BPM para cerveza artesanal, se pregunta por las materias primas e ingredientes, si son debidamente inspeccionados, procesados como sea necesario y almacenados asegurando que sólo se utilicen materiales limpios y sanos. Ellos señalan las siguientes buenas prácticas:

$\checkmark$ Contenedores para almacenamiento sean vaciados y con limpieza rutinaria.

$\checkmark$ Asignar personal para las inspecciones progra-

https://www.brewersassociation.org/wp-content/uploads/2016/03/ GMP-Checklist-Process-and-Controls.pdf madas y de rutina en el almacenamiento.

$\checkmark$ Compra de materias primas e ingredientes con una garantía o certificación del proveedor.

$\checkmark$ Reuniones sobre calidad/inocuidad alimentaria deben llevarse a cabo con proveedores tanto de materias primas como embalajes, a lo menos anualmente.

Con respecto a la tercera "buena práctica" anterior, en las cervecerías encuestadas, en este punto estuvo el mayor porcentaje de deficiencias, en controles para materias primas de cualquier tipo (físico, químico, organoléptico, microbiológico, etc.) el cual puede ser realizado por la cervecería o se puede exigir al proveedor de materias primas para garantizar la calidad higiénica de las mismas. Debido a la importancia de no generar problemas de calidad de la cerveza, los que pueden deberse a materias primas defectuosas, como por ejemplo: cebada contaminada con hongos filamentosos capaces de producir proteínas hidrofobinas que podrían estar presente finalmente en la cerveza y que según Sarlin et al., (2005) citado por Shokribousjein et al., (2011) su presencia es indeseable porque son responsables del brote, es decir, formación de espuma espontánea y salvaje producida inmediatamente después de la apertura del envase sin previa agitación. En el parámetro evaluado ningún establecimiento cumplió totalmente, $59 \%$ de ellos de manera parcial y $41 \%$ sin cumplimiento. Lo anterior indica que en este punto hay que tener mayor control, de hecho, éste debiera ser el primer control dentro del proceso productivo, de manera que sólo se utilicen materias primas en buen estado y se conserven para mantener sus características, que luego serán parte de la cerveza final.

Ítem procesos y productos terminados. Es el ítem con mayor grado de cumplimiento, con un promedio de $73 \%$ y donde los porcentajes fluctuaron entre $11 \%$ y $100 \%$.

En la Figura 9 se observa que 95\% de los establecimientos tuvieron porcentajes de cumplimiento sobre el $45 \%$, y sólo una cervecería obtuvo un notorio bajo porcentaje, a diferencia de sus pares (Cervecería Q, $11 \%)$. En ésta, la mayor falencia estuvo en la falta de una línea de flujo definida, falta de procedimientos escritos y/o instructivos de los procesos de elaboración de cerveza artesanal.

El 95\% de los establecimientos cumple totalmente con el parámetro referente a la distribución final de la cerveza, y el porcentaje restante corresponde a una cervecería donde este parámetro no aplicaba. Lo anterior indica que la distribución se realiza en vehículos autorizados, limpios y en buen estado, siendo este parámetro uno de los mejor evaluados.

Por otra parte, hay algunas deficiencias en otros parámetros. Al respecto, la mayoría de los establecimien- 
tos no contaba con procedimientos escritos, como por ejemplo de formulaciones tanto de productos como de operación y procesos productivos, revelando la ausencia de un sistema de aseguramiento de calidad implementado en ellas, siendo estos procedimientos parte de los requisitos. Existió un 23\% de cumplimiento total del parámetro, indicando una falta de asistencia en lo antes señalado para mejorar el sistema productivo de la cervecería artesanal.

\section{CONCLUSIONES}

No obstante que las industrias cerveceras artesanales encuestadas (73\% de la Región de Los Ríos) no se rigen por lo establecido por el MINSAL de Chile, sino que por la normativa fiscalizada por el SAG, se evaluó el grado de cumplimiento de las BPM en éstas, utilizando como referencia el "Check list" del MINSAL, y se caracterizaron de manera general mediante una pauta adicional.

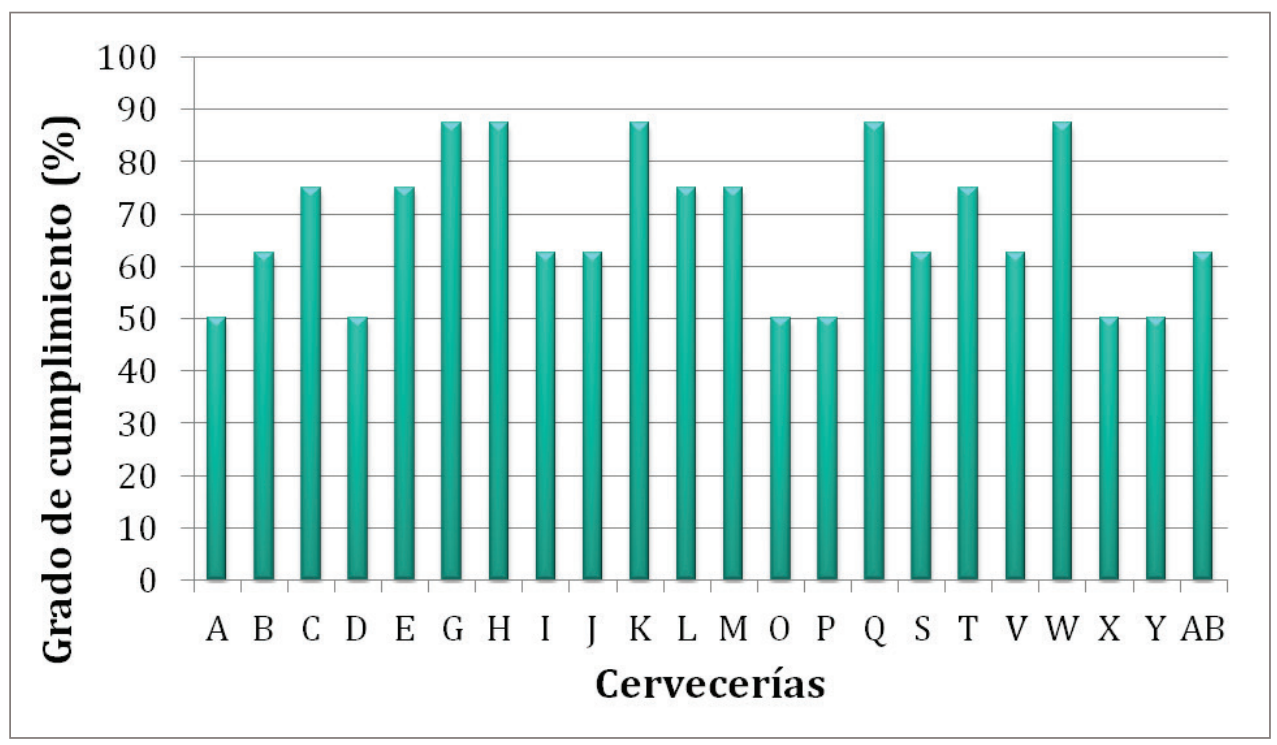

Figura 8. Grado de cumplimiento en el ítem materias primas. Figure 8 . Degree of compliance with the item raw materials.

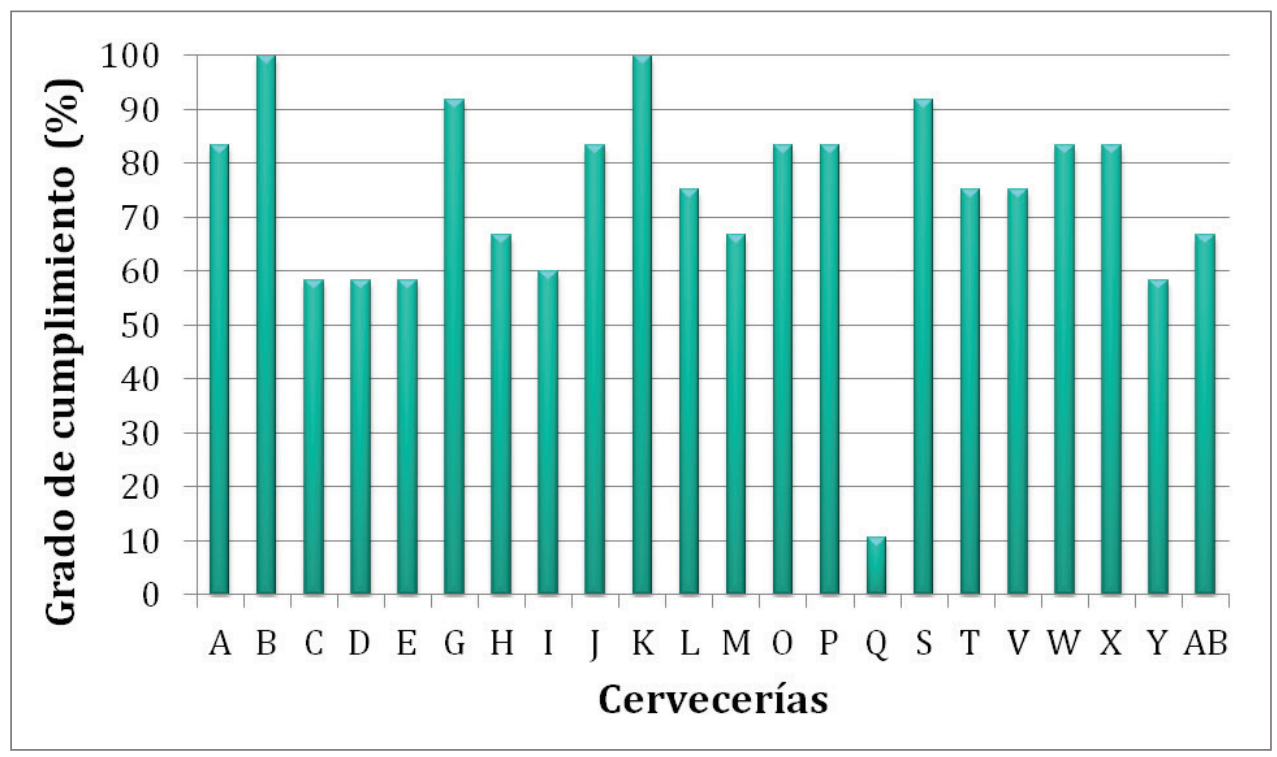

Figura 9. Grado de cumplimiento en el ítem procesos y productos terminados.

Figure 9. Degree of compliance with the item processes and finished products. 
De acuerdo a lo anterior, un bajo porcentaje (sólo un 18\%) de las cervecerías visitadas cumple con las BPM.

El ítem que obtuvo el menor porcentaje de cumplimiento en los establecimientos visitados fue capacitación, arrojando un promedio para todos los establecimientos de sólo un $10 \%$ de cumplimiento. Le siguió higiene del personal y limpieza y sanitización de la planta, cuyos porcentajes no superaron el $50 \%$ de grado de cumplimiento.

El ítem mejor evaluado fue procesos y productos terminados alcanzando en promedio en todos los establecimientos evaluados un $73 \%$ de cumplimiento. Le siguió materias primas con grado de cumplimiento similar.

Se entregaron eficazmente los resultados de la evaluación de BPM, a cada una de las cervecerías visitadas. Recibieron recomendaciones efectivas que ayudan en el cumplimiento de las deficiencias encontradas con respecto a las BPM.

La mayoría de las industrias visitadas se caracterizan por ser Micro cervecerías, según la categorización industrial del Servicio de Impuestos Internos (SII), y por encontrarse distribuidas en varias comunas de la Región de Los Ríos, alrededor de la mitad en zona urbana y la otra parte en la zona rural.

\section{AGRADECIMIENTOS}

A los productores de cerveza artesanal miembros de la Unión de Cerveceros de la Región de Los Ríos (UCR), por su disposición en el período de investigación y visitas en terreno. Al Proyecto FIC-1408 por el respaldo económico y a sus miembros dirigentes por la gentileza al proporcionar información.

\section{REFERENCIAS}

Asociación de Productores de Cerveza de Chile (ACECHI), 2016. Encuesta a cerveceros artesanales. http://www. acechi.cl/home/encuesta-a-cerveceros-artesanales-laclave-para-el-desarrollo-radicaria-en-la-produccionde-cervezas-locales-vinculadas-al-turismo-y-la-gastronomia/ (acceso, 01.08.2016).

Brizio, A., Prentice, C., 2014. Bare or Gloved Hands: A Study on the Production of Safe Food. International Journal of Food Engineering. 10(4), 721-726. http://dx.doi. org/10.1515/ijfe-2014-0196

Cerveceros de España, 2005. Guía para la aplicación del sistema de análisis de peligros y puntos de control crítico en el sector cervecero español. http://www.cerveceros. org/pdf/DocumentoAPPCfinal.pdf (acceso, 13.08.2016)

Chile, Instituto Nacional de Normalización (INN), 2011. Sistema de análisis de peligros y puntos críticos de control (HACCP)-Directrices para su aplicación. NCh 2861.23 p.

Chile, Ministerio de Agricultura (MINAGRI), 2013. Decreto $\mathrm{N}^{\circ} 78$. Ley $\mathrm{N}^{\circ} 18.455$ que fija normas sobre producción, elaboración y comercialización de alcoholes etílicos, bebidas alcohólicas y vinagres. 30 p.

Chile, Ministerio de Salud (MINSAL), 2015. Instructivo aplicación Lista Chequeo BPM. Versión 04. 24 p.

Chile, Servicio Nacional de Turismo (SERNATUR), 2014. Guía de Cervezas Artesanales Región de Los Ríos. Política Regional de Turismo Región de Los Ríos 2011-2014. 20 p.

Chile, Sociedad Chilena de Microbiología e Higiene de los Alimentos (SOCHMHA) y Ministerio de Salud (MINSAL), 2004. Programa de pre-requisitos: Base fundamental para la inocuidad alimentaria. 35 p. http://www.sochmha.cl/documentos/programaprerequisitos.pdf

Di Renzo, J., Casanoves, F., Gonzalez, L., Tablada, E., Díaz, M., Robledo, C., Balzarini, M., 2005. Estadística para las Ciencias Agropecuarias. $6^{\circ}$ ed. Brujas, Córdoba, Argentina.

Hernández, R., Fernández, C.; Baptista, P., 2006. Metodología de la investigación. $4^{\circ}$ ed. MC Graw-Hill, México D.F.

Mancilla, M., 2014. Evaluación de los Riesgos Asociados a Queserías Artesanales de la Región de Los Ríos Mediante la Aplicación de un Instrumento de Buenas Prácticas de Manufactura. Tesis Ingeniero en Alimentos. Universidad Austral de Chile. 38 p

Manzano M., Lacumin L., Vendrame M., Cecchini F., Comi G., Buiatti S., 2011. Craft Beer Microflora Identification Before and After a Cleaning Process. Journal of Institute of Brewing. 117(3), 343-351. http://dx.doi. org/10.1002/j.2050-0416.2011.tb00478.x

Ministerio de Agricultura, Ganadería y Pesca, 2016. Guía de Buenas Prácticas de Manufactura para pequeños establecimientos cerveceros. http://www.alimentosargentinos.gob.ar/contenido/publicaciones/calidad/Guias/ CERVEZAGUIA.pdf (acceso, 01.08.2016).

Paradh, A., Mitchel, W., Hill, A., 2011. Ocurrence of Pectinatus and Megasphaera in the Major UK Breweries. Journal of Institute of Brewing. 117(4), 498-506. http://dx.doi. org/10.1002/j.2050-0416.2011.tb00497.x

Suzuki, K., 2011. 125 th Aniversary Review: Microbiological Instability of Beer Caused by Spoilage Bacteria. Journal of Institute of Brewing. 117(2), 131-155. http://dx.doi. org/10.1002/j.2050-0416.2011.tb00454.x

Shokribousjein Z., Deckers S., Gebruers K., Lorgouilloux Y., Baggerman G., Verachtert H., Delcour J., Etienne P., Rock J., Michiels C., Derdelinckx G., 2011. Hydrophobins, beer foaming and gushing. Cerevisia 35, 85-101. http:// dx.doi.org/10.1016/j.cervis.2010.12.0011 